\title{
Evaluation on mechanical properties of a single trabecular in bovine femur
}

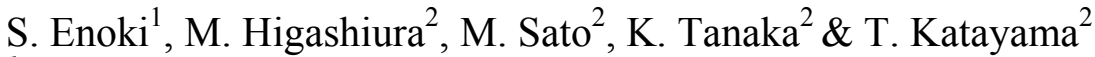 \\ ${ }^{1}$ Department of Mechanical Engineering, \\ Nara National College of Technology, Japan \\ ${ }^{2}$ Department of Biomedical Engineering, Doshisha University, Japan
}

\begin{abstract}
The increase of patients with osteoporosis is a social problem. Osteoporosis decreases bone strength and increases the risk of fracture. The finding of prevention and treatment methods is an urgent issue. Since cancellous bone is metabolically more active than cortical bone, cancellous bone is used for diagnosis of osteoporosis. There are a lot of studies about stress analysis of cancellous bone, and quantities and orientation of trabecular bone that make up the cancellous bone. These studies reported that the trabecular structures contribute to the mechanical properties of cancellous bone. It is considered that the mechanical property of trabecular is based on the assumption that it is constant regardless of the direction and site orientation. However, literature evaluating the mechanical properties of a single trabecular yields a wide dispersion of the results; the mechanical properties of trabecular bone has not been clarified yet. It is necessary to reduce the dispersion of test results to allow for quantitative evaluation of the mechanical properties of trabecular bone and is essential to evaluate the strength of cancellous bone. In this study, single trabecular specimens were polished to bending test specimens in rectangular shape. X-ray $\mu \mathrm{CT}$ was used to obtain shape of trabecular bone specimens. Moreover, three point bending tests were conducted on these specimens and the bending elastic modulus and strength were obtained. Bending elastic modulus and bending strength of the trabecular bone were almost the same value regardless of the direction of each axis. Mechanical properties of trabecular by the bending test do not depend on the orientation of cancellous bone.

Keywords: trabecular, cancellous bone, bending modulus, bending strength, $X$-ray $\mu C T$.
\end{abstract}




\section{Introduction}

Recently, the rising incidence of patients with osteoporosis is becoming a social problem. Osteoporosis decreases bone strength and increases the risk of fracture. Such diseases have a negative impact on quality of life, making it an urgent issue to find its prevention and treatment methods. Patients with osteoporosis are mainly measured by bone mineral density (BMD), such as dualenergy X-ray absorptiometry (DXA) [1], quantitative X-ray computed tomography (QCT) [2] and others. According to the report of National Institute of Health (NIH) in 2000, in order to predict bone strength, bone density alone is not sufficient and it is important to explore other parameters such as "bone quality" serving as an indicator for bone strength measurement [3]. In order to clarify bone quality, it is important to make research on mechanical properties of bone. Bone is a hierarchically structured material and is distinguished into the cortical and cancellous bone. Since cancellous bone is metabolically more active than cortical bone, cancellous bone is used for diagnosis of osteoporosis [4]. There are a lot of studies about stress analysis of cancellous bone, and quantities and orientation of trabecular bone that make up the cancellous bone [5]. These studies reported that the trabecular structures contribute to the mechanical properties of cancellous bone. It is considered that the mechanical property of trabecular is based on the assumption that it is constant regardless of the direction and site orientation. However, literature evaluating the mechanical properties of a single trabecular yields a wide dispersion of the results [6-8]; the mechanical properties of trabecular bone has not been obtained. The cause to these dispersion are considered to be due to the trabecular specimens being obtained and tested without polishing to the proper shape. It is considered that the dispersion in the specimen size cause dispersion in the results of the elastic modulus. The specimen geometry of trabecular had been evaluated in the order from several millimeters to several tens of micrometers by using 2D image of optical microscopy or micrometer. However, since trabecular bone of several hundred micrometers in diameter is a small and irregular in structure, it is considered the shape of the trabecular bone specimen evaluation should be carried out in 3D observation. It is necessary to reduce the dispersion of test results to allow for quantitative evaluation of the mechanical properties of trabecular bone and is essential to evaluate the strength of cancellous bone. In this study, single trabecular specimen were polished to bending test specimens in rectangular shape by using specially designed Jig and X-ray $\mu \mathrm{CT}$ was used to obtain shape of trabecular bone specimens. Three point bending tests were conducted on these specimens and the influence of direction of the trabecular bone specimen on the bending elastic modulus and strength were discussed. 


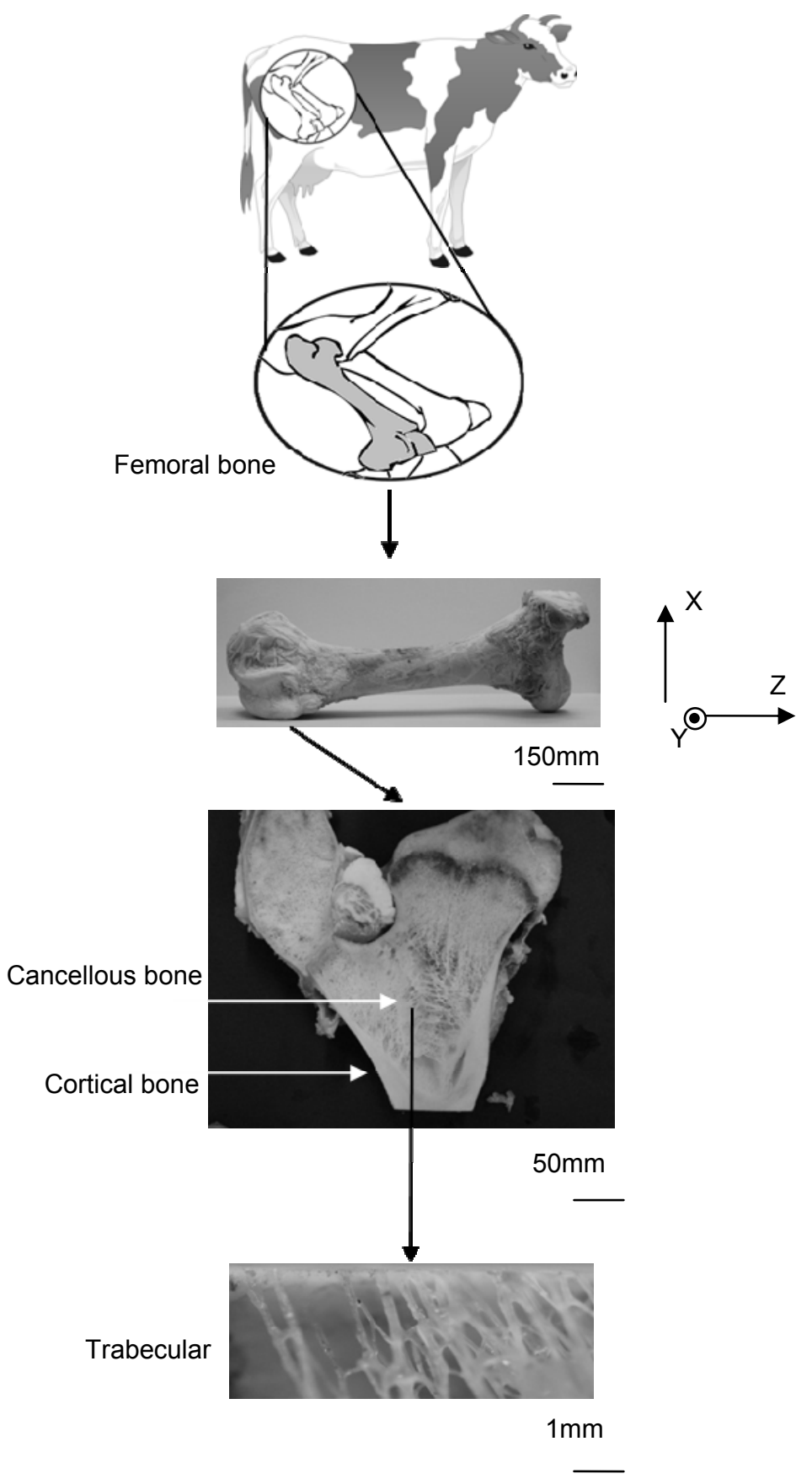

Figure 1: Trabecula on each axis obtained from the right distal femur of a bovine. 


\section{Materials and methods}

\subsection{Specimen preparation}

Trabecular bones were obtained from cancellous bones of a bovine femur, at the $\mathrm{Z}$-axis direction in parallel to the major axis of the femur, the $\mathrm{X}$-axis and $\mathrm{Y}$-axis direction in perpendicular to the major axis of the femur as indicated in fig. 1 . Single trabecular specimen on each axis was extracted from the cancellous bone in length of $2 \mathrm{~mm}$ to $3 \mathrm{~mm}$ and in diameter of $200 \mu \mathrm{m}$ to $500 \mu \mathrm{m}$. Trabecular bone has been processed into a rectangular. Trabecular bone was polished to the thickness of the trabecular bone to $100 \mu \mathrm{m}$ as shown in fig. 2 by using Speed Lap (Maruto ML-521-d). Speed Lap is a double-sided polishing machine flake type automatic lap polishing to create a slice. Side polishing was performed by using the sandpaper (600grit) to form the width of $250 \mu \mathrm{m}$ of trabecular bone. Cantilever jig (Jig A, shown in fig.3) [9] and Both ends supported jig (Jig B, shown in Fig.4) were used to support specimens duting polishing. Three dimension images of each trabecular bone specimen (3D images) was obtained by using micro focus X-ray system (Shimadzu, SMX-160CTS). Width and thickness of specimens were obtained from the average value of three points of 3D image.
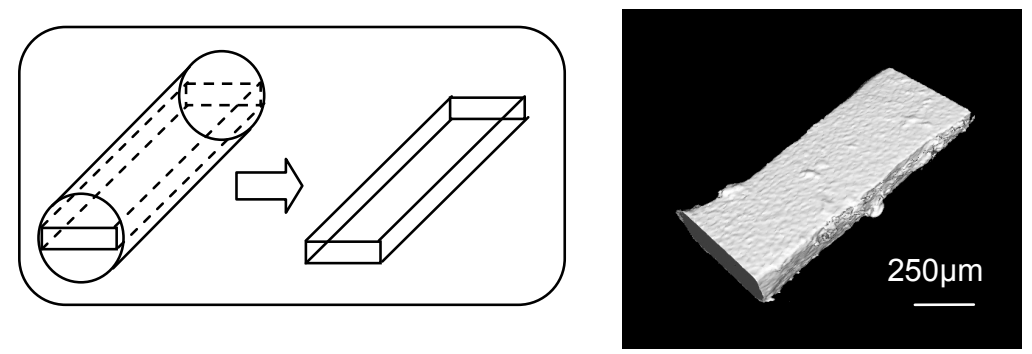

Figure 2: $\quad \mathrm{X}$ ray $\mu \mathrm{CT}$-imaged of trabecular bone specimen after speed lapping.

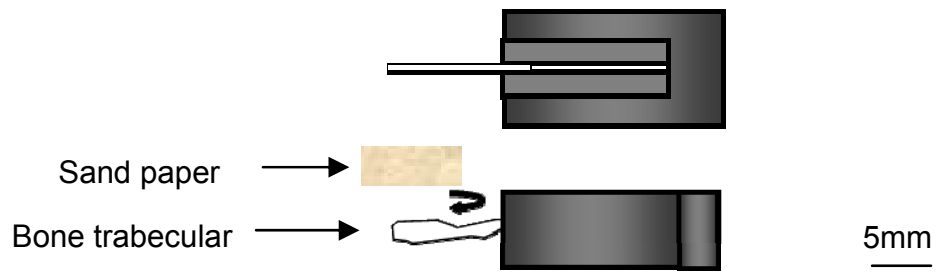

Figure 3: $\quad$ Cantilever jig (Jig A). 


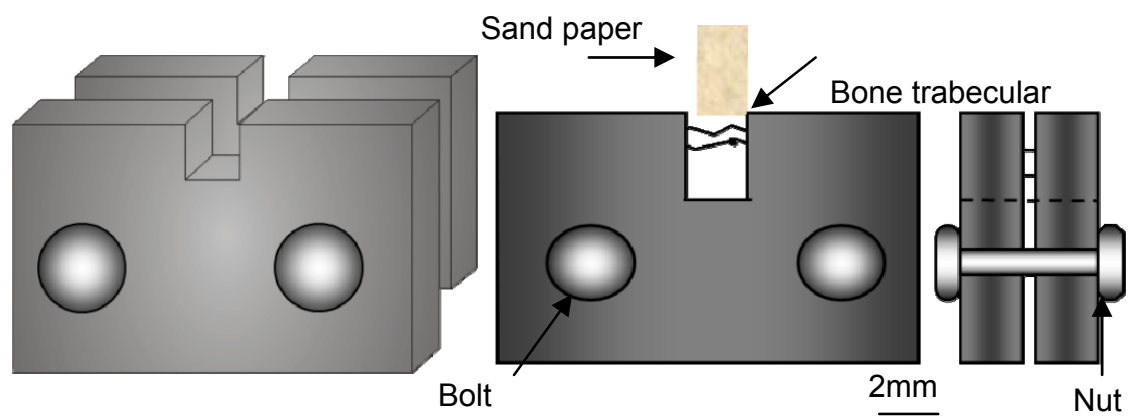

Cabinet projection drawing

Front view

Side view

Figure 4: Both ends supported jig (Jig B).

\subsection{Bending test of trabecular}

Elastic bending tests were performed by using Micro Material Tester (Shimadzu, MMT-11NV-2) with a load cell capacity of $10 \mathrm{~N}$. Each specimen was placed on the jig as shown in fig. 5 and span length was set to $1.1 \mathrm{~mm}$. Knife-edge indenter of $0.1 \mathrm{~mm}$ in tip radius was used as an indenter within the three point bending test. The positioning of specimen was conducted by XY stage in resolution of $0.01 \mathrm{~mm}$. Elastic bending test was carried out at a crosshead speed of $0.001 \mathrm{~mm} / \mathrm{sec}$, and maximum displacement was $0.02 \mathrm{~mm}$. The following equation [10] was used to calculate the elastic modulus for each specimen.

$$
E=\frac{(P / \delta) L^{3}}{4 b h^{3}}\left[1+2.85(h / L)^{2}-0.84(h / L)^{3}\right]
$$

$E$ : Elastic modulus

$P / \delta$ : Load displacement curve

$L$ : Distance between the fulcrum

$b$ : Width of the specimen

$h$ : Thickness of the specimen

Bending strength test was performed and carried out at a crosshead speed of $0.001 \mathrm{~mm} / \mathrm{sec}$, and maximum displacement was $1 \mathrm{~mm}$. The following equation was used to calculate the bending stress for each specimen.

$b_{e:}$ Bending strength

$$
\sigma_{b}=\frac{M}{I} \times \frac{h}{2}=\frac{P L / 4}{b h^{3} / 12} \times \frac{h}{2}
$$

M: Maximum bending moment

I: Second moment of area

$P$ : Load

$L$ : Distance between the fulcrum

$b$ : Width of the specimen

$h$ : Thickness of the specimen. 


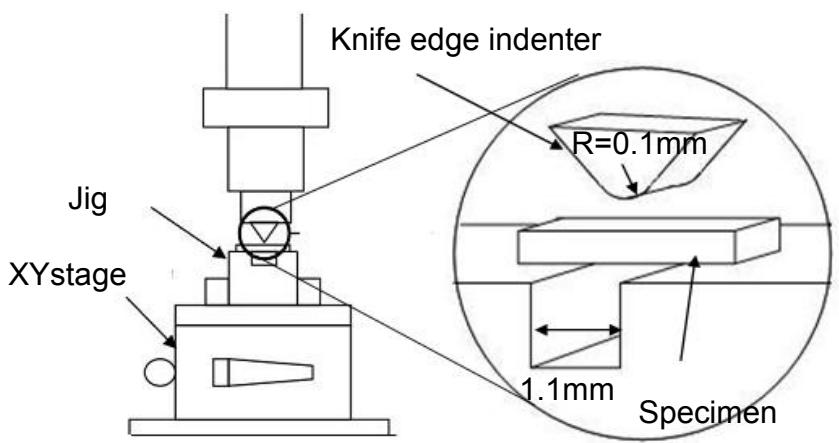

Figure 5: $\quad$ Three point bending tester.

\section{Results and discussion}

\subsection{Influence of supporting jig during polishing process on specimen dimensions and bending tests}

Figs. 6 and 7 show the cross-sectional, and the overall view of the specimens which were prepared by using Jig A and Jig B described in Section 2.1. Twenty trabecular bone specimens obtained from the Y-axis direction were used. The side of the specimens shown in fig. 7 has been polished more uniformly than fig.6. Fig.8 shows a comparison of the width dimension of the specimens polished by Jig A and Jig B. According to fig. 8 width dimension by using Jig A yields $256 \pm 22.7 \mu \mathrm{m}$ with relative standard deviation at $8.87 \%$; meanwhile width dimension by Jig B yields $250 \pm 7.73 \mu \mathrm{m}$ and relative standard deviation at $3.09 \%$. Jig B can polish the side of the specimens better than Jig A. Fig.9 shows the bending results to specimens polished by using Jig A and Jig B. The elastic modulus of the specimens by Jig A yields $7.58 \pm 1.96 \mathrm{GPa}$, with relative standard deviation at 25.9\%; meanwhile the elastic modulus by Jig B yields $7.77 \pm$ $0.765 \mathrm{GPa}$ with relative standard deviation at $9.85 \%$. Relative standard deviation

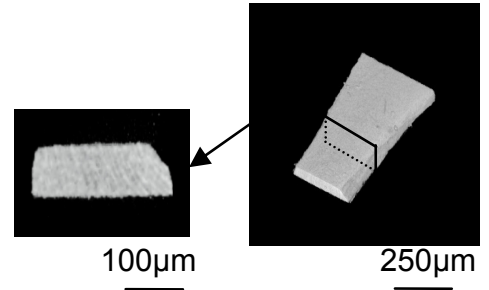

Figure 6: Trabecula specimen polished by using Jig A.

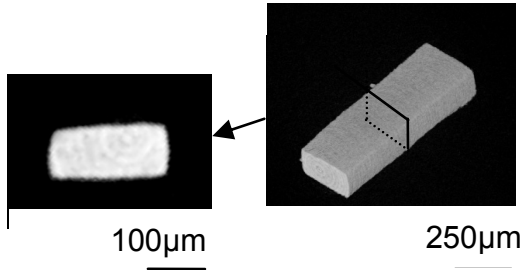

Figure 7: Trabecula specimen polished by using Jig B. 
for the specimens by Jig B has decreased $16.1 \%$ compared to Jig A. The accuracy of the width dimensions improved that of elastic modulus. By using the both ends supported jig (Jig B), the bending elastic modulus can be obtained with small standard deviation.

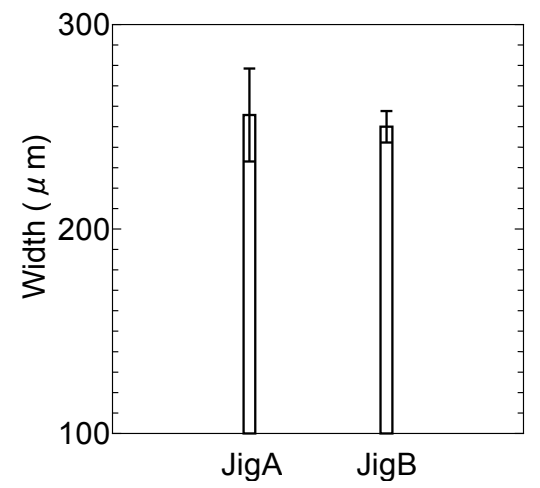

Figure 8: Width of specimen polished by using Jig A and Jig B.

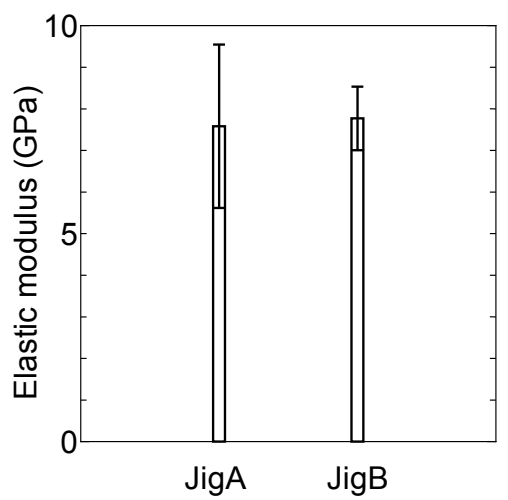

Figure 9: Elastic modulus of specimen polished by using Jig A and Jig B.

\subsection{Bending test}

All specimens were prepared by using the Jig B. Fig.10 shows the comparison of the elastic modulus of ten trabecular bone specimens obtained from each of the $\mathrm{X}, \mathrm{Y}$ and $\mathrm{Z}$ axis. Elastic modulus of $\mathrm{X}, \mathrm{Y}$ and $\mathrm{Z}$ axis was $7.42 \pm 0.657 \mathrm{GPa}$, $7.77 \pm 0.765 \mathrm{GPa}$ and $7.45 \pm 0.861 \mathrm{GPa}$ respectively. There was no significant difference in the elastic modulus. It is estimated that the bending elastic modulus of trabecular bone does not depend on the orientation of cancellous bone. Fig. 11 shows the comparison of the elastic strength of ten trabecular bone specimens obtained from each of the $\mathrm{X}, \mathrm{Y}$ and $\mathrm{Z}$ axis. Bending strength of $\mathrm{X}, \mathrm{Y}$ and $\mathrm{Z}$ axis was $247 \pm 17.1 \mathrm{MPa}, 251 \pm 14.8 \mathrm{MPa}$ and $247 \pm 16.2 \mathrm{MPa}$ respectively. There was no significant difference in bending strength. It is estimated that the bending strength of trabecular bone does not depend on the orientation of cancellous bone. On the other hand, it was reported that for compression test in the $\mathrm{X}, \mathrm{Y}$ and $\mathrm{Z}$ axis direction of the cancellous bone, there was a significant difference to elastic modulus and to the orientation of the trabecular bone that make up the cancellous bone [9]. According to the reported results and our study, structural parameters of trabecular bone such as thickness, weight, orientation and connectivity rather than characteristics of the trabecular is related to the anisotropy of the cancellous bone structure. 


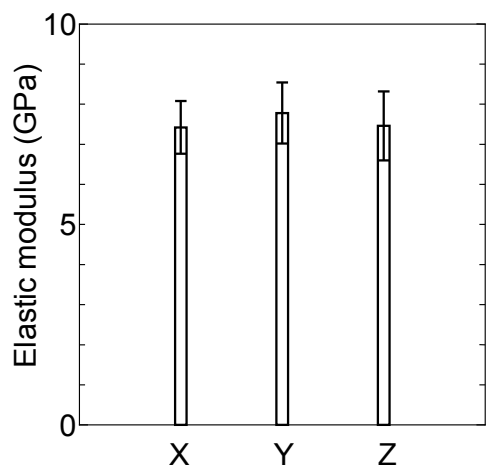

Figure 10: Elastic modulus of trabecula specimen on each $\mathrm{X}, \mathrm{Y}$, and $\mathrm{Z}$ axis obtained from elastic bending test.

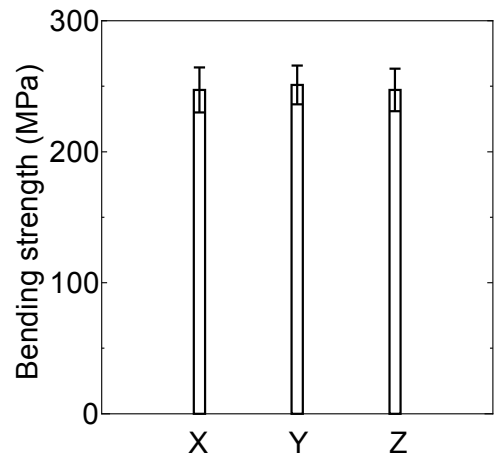

Figure 11: Bending strength of trabecula specimen on each $\mathrm{X}, \mathrm{Y}, \mathrm{Z}$ axis.

\section{Conclusions}

Single trabecular samples were polished to bending test specimens in rectangular shape by using specially designed Jig and X-ray $\mu \mathrm{CT}$ was used to obtain shape of trabecular bone specimens. Three point bending tests were conducted on these specimens and the influence of direction of the trabecular bone specimen on the bending elastic modulus and strength were discussed. The investigation yields the following conclusions.

1. By using the both ends supported jig, the bending elastic modulus and strength were obtained with small standard deviation.

2. There were no significant difference in the elastic modulus and strength of the trabecular bones which were extracted in parallel and in perpendicular to the major axis of a femur. 


\section{References}

[1] Kanis, J.A., Delmas, P., Burckhardt, P., Cooper, C., Torgerson, D. Guidelines for diagnosis and management of osteoporosis, Osteoporosis International 7, pp.390-406, 2009.

[2] Guglielmi, G. Quantitative computed tomography (QCT) and dual X-ray absorptiometry, (DXA) in the diagnosis of osteoporosis European Journal of Radiology 20, pp. 185-187, 1995.

[3] Mary L, Bouxsein, Bone quality, where do we go from here? Osteoporosis International Volume 14, Supplement 5, 2003.

[4] Parfitt, A.M., Misconceptions (2): Turnover is always higher in cancellous than in cortical bone, bone 30 (6), pp. 807-809, 2002.

[5] Morgan, E.F., Keaveny, T.M., Dependence of yield strain of human trabecular bone on anatomic site, Journal of Biomechanics 34 (5), pp. 569577, 2001.

[6] Ito, M., Nishida, A., Koga, A., Ikeda, S., Shiraishi, A., Uetani, M., Hayashi, K., Nakamura, T., Contribution of trabecular and cortical components to the mechanical properties of bone and their regulating parameters, Bone 31 (3), pp. 351-358, 2002.

[7] Tsubota, K., Adachi, T., Nishiumi, S., Tomita, Y., Elastic properties of single trabeculae measured by micro-three-point bending test, ATEM'03, JSME-MMD, Sep. 10-12, 2003.

[8] Jungmann, R., Schitter, G., Fantner, G.E., Lauer, M.E., Hansma, P.K. and Thurner, P.J., Real-time microdamage and strain detection during micromechanical testing of single trabeculae, Experimental and Applied Mechanics: SEM Annual Conference and Exposition, pp.11, 2007.

[9] Enoki, S., Sato, M., Tanaka, K., Katayama, T., Mechanical properties of a single cancellous bone trabeculae taken from bovine femur, International Journal of Modern Physics: Conference Series (IJMPCS), 2011.

[10] Timoshenko. S. P., Theory of elasticity, Corona Publishing Co., Ltd pp. 117-125, 1973. 